Article

\title{
Analysis of the Association Between MicroRNA Biogenesis Gene Polymorphisms and Venous Thromboembolism in Koreans
}

\author{
Eun Ju Ko ${ }^{1,+}$, Eo Jin Kim ${ }^{2,+}$, Jung Oh Kim ${ }^{1}$, Jung Hoon Sung ${ }^{3}$, Han Sung Park ${ }^{1}$, \\ Chang Soo Ryu ${ }^{1}$, Jisu Oh ${ }^{3}$, So Young Chong ${ }^{3}$, Doyeun $\mathrm{Oh}^{3}$ and Nam Keun Kim ${ }^{1, *} \mathbb{E}$ \\ 1 Department of Biomedical Science, College of Life Science, CHA University, Seongnam 13488, Korea \\ 2 Department on Internal Medicine, Asan Medical Center, University of Ulsan College of Medicine, \\ Seoul 05505, Korea \\ 3 Department of Internal Medicine, CHA Bundang Medical Center, CHA University, Seongnam 13496, Korea \\ * Correspondence: nkkim@cha.ac.kr; Tel.: +82-31-881-7137 \\ + These authors contributed equally to this work.
}

Received: 2 July 2019; Accepted: 31 July 2019; Published: 1 August 2019

\begin{abstract}
Venous thromboembolism (VTE) involves the formation of a blood clot, typically in the deep veins of the leg or arm (deep vein thrombosis), which then travels via the circulatory system and ultimately lodges in the lungs, resulting in pulmonary embolism. A number of microRNAs (miRNAs) are well-known regulators of thrombosis and thrombolysis, and mutations in miRNA biogenesis genes, such as DICER1, DROSHA have been implicated in miRNA synthesis and function. We investigated the genetic association between polymorphisms in four miRNA biogenesis genes, DICER1 rs3742330A > G, DROSHA rs10719T > C, RAN rs14035C > T and XPO5 rs11077A > C, and VTE in 503 Koreans: 300 controls and 203 patients. Genotyping was assessed with polymerase chain reaction-restriction fragment length polymorphism assays. We detected associations between polymorphisms in $R A N$ and XPO5 and VTE prevalence ( $R A N$ rs14035CC + CT versus TT: $p=0.018 ;$ XPO5 rs11077AA + AC versus CC: $p<0.001$ ). Analysis of allele combinations of all four polymorphisms (DICER1, DROSHA, RAN, XPO5) revealed that A-T-T-A was associated with decreased VTE prevalence $(p=0.0002)$, and A-T-C-C was associated with increased VTE prevalence $(p=0.027)$. Moreover, in subjects with provoked VTE, the DROSHA rs10719T > C, polymorphism was associated with increased disease prevalence (TT versus $\mathrm{TC}+\mathrm{CC}: p<0.039$ ). Our study demonstrates that RAN and XPO5 polymorphisms are associated with risk for VTE in Korean subjects.
\end{abstract}

Keywords: vascular diseases; venous thromboembolism; microRNAs; 3' untranslated regions; polymorphism; single nucleotide

\section{Introduction}

Venous thromboembolism (VTE), which involves deep vein thrombosis (DVT) followed by pulmonary embolism (PE), is the third most common cardiovascular disease following myocardial infarction and stroke [1]. DVT is a disease that occurs when blood clots form in the deep veins, usually in the legs. The thrombus can then become dislodged and migrate to form a blockage in the pulmonary veins, with $45.4 \%$ of DVT patients ultimately developing PE [2,3]. The incidence of VTE is higher in western populations than in Asians, although recently, annual increases in the incidence of VTE have been noted in Asian individuals, reaching levels as high as that of western people under certain circumstances [4,5]. VTE can be classified into two groups, known as provoked and unprovoked [6]. The provoked group includes patients with a known cause of a hypercoagulable state, including female hormones (estrogens or oral contraception), surgery (post-operative period, particularly for orthopedic 
surgery of the hip or knee, or urinary surgery), pregnancy, phospholipid antibodies in the blood (e.g., anti-cardiolipin antibodies or lupus anticoagulant), cancer, high levels of blood homocysteine, or inherited protein deficiencies associated with coagulation [2,7]. In contrast, unprovoked VTE occurs in patients with no clear causality. Pro-clinical symptoms of DVT include swelling, redness, tenderness of the leg, and manifestations of PE, such as syncope, wheezing, substernal pain, hemoptysis, tachycardia, or tachypnea [8]. However, because these symptoms are not specific to VTE, only about $20 \%$ of patients with DVT or PE are properly diagnosed $[2,9,10]$.

The underlying causes of thrombosis are referred to as Virchow's Triad; these include endothelial injury, abnormal blood flow, and hypercoagulation [8]. Notably, a previous study found that microRNAs (miRNAs) are associated with hemostasis [11] and are involved in the formation of blood clots, the hypercoagulable state, and endothelial cell proliferation [12-16]. These miRNAs species are short, non-coding RNAs, 22-24 nucleotides in length, which control gene expression as post-transcriptional regulators. The miRNA-encoding genes are transcribed by RNA polymerase II as long, primary miRNAs (pri-miRNA) with a stem-loop structure (100-1000 nts), that are cleaved by the DROSHA/DGCR8 complex in nucleus. The resulting 80-nt, hairpin-shaped precursor miRNAs (pre-miRNAs) are exported to the cytoplasm by the Ras-related nuclear protein (RAN) GTPase and exportin 5 (XPO5). There, they are processed by the DICER1/TRBP complex to form miRNA duplex. One strand of the miRNA duplex is loaded onto Argonaute (AGO) to form the RNA-induced silencing complex (RISC), which binds to the $3^{\prime}$-untranslated region (UTR) of an mRNA transcript and functions to suppress translation or promote mRNA degradation, through a mechanism known as post-transcriptional gene silencing (PTGS) [17-19]. Regulation by miRNA-mediated PTGS plays a fundamental role in many essential cellular processes, and because of this, proper miRNA biogenesis is critical for maintaining metabolic balance [20-22].

The miRNA biogenesis genes are critical for miRNA synthesis, and mutations in these genes can have global effects on miRNA expression [23-27]. For example, smoking-induced damage to the gene encoding DICER1 results in altered alveolar macrophage miRNA expression [28]. Mutations in DROSHA are associated with effects on male fertility, and in particular, a lack of DROSHA in the male germline causes defects in miRNA production and spermatogenesis [29]. In addition, mutations in XPO5 have been implicated in the development and progression of certain cancers. Specifically, in hepatocellular carcinoma, a mutant form of this protein is aberrantly phosphorylated by extracellular signal-regulated kinase (ERK) kinase, undergoes a conformational change, and loses the ability to transport precursor miRNAs. As a result, pre-miRNA is retained in the nucleus, and global miRNA expression levels are down-regulated [30].

A number of studies have also shown that miRNAs play important roles conditions associated with increased risk for VTE, such as diabetes mellitus, hypertension, and hypercholesterolemia [31-33], as well as in thrombosis development directly. One previous study, in particular, revealed that miRNA-145 is associated with thrombus formation, with lower levels present in VTE patients. This miRNA represses expression of tissue factor (TF), and it is proposed that miRNA-145-mediated down-regulation of TF is required for proper function of the intrinsic coagulation pathway and the suppression of thrombus production [14]. Several other miRNAs are also associated with various aspects of vascular biology and hemostasis, including platelet biogenesis (megakaryopoiesis) and function, expression of coagulation factor and anticoagulation factor, and fibrinolysis. For example, miRNA-23 and miRNA-27 are associated with pro-angiogenesis functions, whereas miRNA-17, miRNA-92, and miRNA-32 are involved in anti-angiogenesis. In addition, miRNA-223 plays an important role in platelet formation and thrombin activation, and both miRNA-19b and miRNA-20a are associated with pro-coagulant activity in anti-phospholipid syndrome (APS) and systemic lupus erythematous (SLE) patients $[15,16]$.

In our previous study, we detected an association between $3^{\prime}$-UTR polymorphisms in miRNA biogenesis genes and ischemic stroke risk and prognosis. In particular, polymorphisms at DICER1 rs3742330 and DROSHA rs10719 were found to be associated with ischemic stroke 
susceptibility, and the RAN rs14035 polymorphic locus was significantly linked to post-stroke mortality [34]. Based on these findings, in this study, we selected four genes (DICER1, DROSHA, $R A N, X P O 5)$ among several miRNA biogenesis genes. The DROSHA gene is located on 15p13.3 and DROSHA is a nuclear ribonulcease III (RNase III) enzyme, which cleaves a stem-loop pri-miRNAs to hairpin-shaped pre-miRNAs. DICER1 gene is located on 14q32.13 and DICER1 is another RNase III, which cleaves the pre-miRNA to intermediate miRNA duplex [35]. RAN gene is located on 12q24.33 and RAN is a GTPase that makes a pre-miRNA/XPO5/Ran-GTP ternary complex and transports pre-miRNA to cytoplasm. XPO5 gene is located on 6p21.1 and XPO5 is a member of the karyopherin family and exports pre-miRNAs from the nucleus to cytoplasm [36]. Moreover, we investigated 3'-UTR polymorphisms of this four miRNA biogenesis gene, including DICER1 rs3742330A > G, DROSHA rs10719T $>$ C, RAN rs14035C > T, and XPO5 rs11077A $>$ C, which are independently or complementarily associated with disease prevalence and clinical outcomes in VTE patients.

\section{Results}

\subsection{Characteristics of the Study Population.}

The demographic characteristics for VTE patients and controls are listed in Table 1. There were no significant differences in age, hypertension, hyperlipidemia, or smoking status between the two groups. In contrast, VTE was more prevalent among men, and the patient group included significantly more subjects with diabetes.

Table 1. Baseline characteristics of venous thromboembolism (VTE) patients and control subjects.

\begin{tabular}{|c|c|c|c|c|c|}
\hline Characteristic & $\begin{array}{l}\text { Controls } \\
(n=300)\end{array}$ & $\begin{array}{l}\text { VTE Patients } \\
(n=203)\end{array}$ & $p$-value ${ }^{a}$ & $\begin{array}{l}\text { Unprovoked VTE } \\
(n=93)\end{array}$ & $p$-value ${ }^{\mathrm{a}}$ \\
\hline Age (years, mean \pm SD) & $57.18 \pm 9.96$ & $56.07 \pm 17.79$ & 0.881 & $57.23 \pm 17.76$ & 0.447 \\
\hline Male (\%) & $138(46.0)$ & $103(50.7)$ & 0.297 & $54(58.1)$ & 0.042 \\
\hline Hypertension (\%) & $94(31.3)$ & $61(30.0)$ & 0.760 & $31(33.3)$ & 0.718 \\
\hline DM (\%) & $26(8.7)$ & $31(15.3)$ & 0.022 & $17(18.3)$ & 0.010 \\
\hline Lipidemia (\%) & $51(17.0)$ & $41(20.2)$ & 0.363 & $16(17.2)$ & 0.964 \\
\hline Smoking (\%) & $104(34.7)$ & $58(28.6)$ & 0.152 & $33(35.5)$ & 0.885 \\
\hline
\end{tabular}

\subsection{Genotype Frequencies of miRNA Biogenesis Genes}

We investigated the prevalence of the DICER1 rs3742330A > G, DROSHA rs10719T > C, RAN rs14035C $>$ T, and XPO5 rs11077A $>$ C gene polymorphisms in VTE patients and control subjects. Table 2 shows the genotype distributions in each of these groups. We found that the XPO5 rs11077A > $C$ polymorphism was associated with an increased risk for VTE (AA versus AC: adjusted odds ratio $(\mathrm{AOR})=2.522,95 \%$ confidence interval $(\mathrm{CI})=1.564-4.067, p<0.001 ; \mathrm{AA}$ versus $\mathrm{AC}+\mathrm{CC}$ : $\mathrm{AOR}=2.493$, $95 \% \mathrm{CI}=1.552-4.003, p<0.001)$. In contrast, the RAN CT genotype was more frequent in control group than VTE patients, and the RAN rs14035C > T polymorphism was associated with reduced VTE risk (CC versus $\mathrm{CT}$ : $\mathrm{AOR}=0.630,95 \% \mathrm{CI}=0.425-0.935, p=0.022 ; \mathrm{CC}$ versus $\mathrm{CT}+\mathrm{TT}$ : $\mathrm{AOR}=0.627$, $95 \% \mathrm{CI}=0.427-0.922, p=0.018$ ). The XPO5 $\mathrm{rs} 11077 \mathrm{~A}>\mathrm{C}$ polymorphism showed the same increased AOR pattern in subjects with both provoked and unprovoked VTE, whereas the RAN rs14035C $>\mathrm{T}$ polymorphism also demonstrated a reduced AOR in subjects with unprovoked VTE. The DICER1 rs3742330A > G and DROSHA rs10719T > C polymorphisms were not significantly correlated with either total or unprovoked VTE occurrence. However, for provoked VTE, the DROSHA rs10719T > C polymorphism was associated with an increased disease risk (TT versus TC + CC: AOR $=2.460,95 \%$ $\mathrm{CI}=1.048-5.774, p=0.039$ ). 
Table 2. Genotype frequencies of DICER1, DROSHA, RAN, and XPO5 polymorphisms in VTE patients and controls.

\begin{tabular}{|c|c|c|c|c|c|c|c|c|c|c|}
\hline Genotype & $\begin{array}{l}\text { Controls } \\
(n=300)\end{array}$ & $\begin{array}{l}\text { Total VTE } \\
(n=203)\end{array}$ & AOR $(95 \% \mathrm{CI})^{\mathrm{a}}$ & $p$-value ${ }^{\mathrm{b}}$ & $\begin{array}{c}\text { Unprovoked VTE } \\
(n=93)\end{array}$ & AOR $(95 \% \mathrm{CI})^{\mathrm{a}}$ & $p$-value ${ }^{b}$ & $\begin{array}{c}\text { Provoked VTE } \\
\quad(n=110)\end{array}$ & $\operatorname{AOR}(95 \% \mathrm{CI})^{\mathrm{a}}$ & $p$-value ${ }^{\mathrm{b}}$ \\
\hline \multicolumn{11}{|l|}{ DICER1 } \\
\hline \multicolumn{11}{|l|}{ rs $3742330 A>G$} \\
\hline AA & $109(36.3)$ & $79(38.9)$ & 1.000 (reference) & & $42(45.2)$ & 1.000 (reference) & & & 1.000 (reference) & \\
\hline AG & 137 (45.7) & $92(45.3)$ & $0.953(0.638-1.424)$ & 0.815 & $35(37.6)$ & $0.685(0.403-1.164)$ & 0.162 & $37(33.6)$ & $1.247(0.756-2.057)$ & 0.387 \\
\hline GG & $54(18.0)$ & $32(15.8)$ & $0.788(0.460-1.350)$ & 0.386 & $16(17.2)$ & $0.729(0.371-1.432)$ & 0.359 & $57(51.8)$ & $0.830(0.413-1.669)$ & 0.602 \\
\hline Dominant & & & $0.919(0.633-1.336)$ & 0.659 & & $0.708(0.438-1.144)$ & 0.158 & $16(14.5)$ & $1.130(0.704-1.815)$ & 0.612 \\
\hline Recessive & & & $0.873(0.536-1.423)$ & 0.586 & & $0.971(0.519-1.815)$ & 0.926 & & $0.765(0.411-1.425)$ & 0.399 \\
\hline HWE $p$-value & 0.342 & 0.547 & & & & & & & & \\
\hline \multicolumn{11}{|l|}{ DROSHA } \\
\hline \multicolumn{11}{|l|}{ rs10719T $>C$} \\
\hline TT & $164(54.7)$ & $116(57.1)$ & 1.000 (reference) & & $50(53.8)$ & 1.000 (reference) & & $66(60.0)$ & 1.000 (reference) & \\
\hline TC & $123(41.0)$ & $72(35.5)$ & $0.892(0.608-1.309)$ & 0.559 & $39(41.9)$ & $1.101(0.673-1.799)$ & 0.703 & $33(30.0)$ & $0.696(0.426-1.136)$ & 0.147 \\
\hline CC & $13(4.3)$ & $15(7.4)$ & $1.893(0.837-4.278)$ & 0.125 & $4(4.3)$ & $1.250(0.379-4.120)$ & 0.714 & $11(10.0)$ & $2.200(0.891-5.428)$ & 0.087 \\
\hline Dominant & & & $0.970(0.672-1.400)$ & 0.871 & & $1.096(0.680-1.766)$ & 0.707 & & $0.828(0.525-1.306)$ & 0.418 \\
\hline Recessive & & & $1.897(0.872-4.127)$ & 0.106 & & $1.115(0.349-3.563)$ & 0.854 & & 2.460 (1.048-5.774) & 0.039 \\
\hline HWE $p$-value & 0.089 & 0.414 & & & & & & & & \\
\hline \multicolumn{11}{|l|}{ RAN } \\
\hline \multicolumn{11}{|l|}{ rs $14035 C>T$} \\
\hline $\mathrm{CC}$ & $178(59.3)$ & 141 (69.5) & 1.000 (reference) & & $71(76.3)$ & 1.000 (reference) & & $70(63.6)$ & 1.000 (reference) & \\
\hline $\mathrm{CT}$ & 113 (37.7) & $58(28.6)$ & $0.630(0.425-0.935)$ & 0.022 & $21(22.6)$ & $0.433(0.248-0.756)$ & 0.003 & $37(33.6)$ & $0.833(0.519-1.338)$ & 0.450 \\
\hline TT & $9(3.0)$ & $4(2.0)$ & $0.569(0.169-1.919)$ & 0.363 & $1(1.1)$ & $0.268(0.032-2.217)$ & 0.222 & $3(2.7)$ & $0.973(0.249-3.796)$ & 0.969 \\
\hline Dominant & & & $0.627(0.427-0.922)$ & 0.018 & & $0.423(0.245-0.730)$ & 0.002 & & $0.842(0.531-1.337)$ & 0.467 \\
\hline HWE $p$-value & 0.073 & 0.482 & & & & & & & & \\
\hline \multicolumn{11}{|l|}{ XPO5 } \\
\hline \multicolumn{11}{|l|}{ rs11077A $>C$} \\
\hline AA & $263(87.7)$ & $148(72.9)$ & 1.000 (reference) & & $67(72.0)$ & 1.000 (reference) & & $81(73.6)$ & 1.000 (reference) & \\
\hline AC & 36 (12.0) & $54(26.6)$ & $2.522(1.564-4.067)$ & $<0.001$ & $25(26.9)$ & $2.611(1.450-4.700)$ & 0.001 & $29(26.4)$ & 2.398 (1.359-4.233) & 0.003 \\
\hline $\mathrm{CC}$ & $1(0.3)$ & $1(0.5)$ & $1.542(0.093-25.601)$ & 0.763 & $1(1.1)$ & $2.971(0.175-50.478)$ & 0.451 & $0(0.0)$ & - & - \\
\hline Dominant & & & $2.493(1.552-4.003)$ & $<0.001$ & & $2.624(1.469-4.689)$ & 0.001 & & $2.336(1.326-4.116)$ & 0.003 \\
\hline Recessive & & & $1.119(0.068-18.398)$ & 0.937 & & $2.516(0.150-42.109)$ & 0.521 & & - & - \\
\hline HWE $p$-value & 0.843 & 0.091 & & & & & & & & \\
\hline
\end{tabular}

${ }^{a}$ Adjusted by age, gender, hypertension, diabetes mellitus, lipidemia, and smoking status. ${ }^{\mathrm{b}} p$-values were calculated using logistic regression analysis. Significant $p$-values $<0.05$ are shown in bold. Abbreviations: AOR, adjusted odds ratio; $95 \%$ CI, 95\% confidence interval; HWE, Hardy-Weinberg equilibrium; VTE, venous thromboembolism. 


\subsection{Genotype Combinations of miRNA Biogenesis Gene Polymorphisms}

We next performed genotype combination analyses for the miRNA biogenesis gene polymorphisms tested in this study (Table 3). These data revealed that the RAN rs14035 CC/XPO5 rs11077 AC genotype was significantly more frequent in VTE patients than in control subjects (AOR $=2.061,95 \%$ $\mathrm{CI}=1.153-3.686$, FDR-adjusted $p=0.045$ ). Similarly, the DICER1 rs3742330 AA/XPO5 rs11077 AC and DROSHA rs10719 TT/XPO5 rs11077 AC genotypes were more frequently observed in VTE patients $(\mathrm{AOR}=4.326,95 \% \mathrm{CI}=2.024-9.245$, FDR-adjusted $p=0.001 ; \mathrm{AOR}=2.385,95 \% \mathrm{CI}=1.239-4.590$, FDR-adjusted $p=0.047$, respectively). In contrast, the frequencies of the RAN rs14035 CT/XPO5 rs 11077 AA (AOR $=0.583,95 \% \mathrm{CI}=0.373-0.912$, FDR-adjusted $p=0.045)$ and DROSHA rs10719 TT/RAN rs14035 CT (AOR $=0.445,95 \% \mathrm{CI}=0.250-0.790$, FDR-adjusted $p=0.040)$ genotypes were significantly higher in control participants.

We also performed genotype combination analysis in unprovoked VTE patients (Table 3) and found that the DICER1 rs3742330 AA/XPO5 rs11077 AC genotypes were more frequently observed in VTE patients (AOR $=4.709,95 \% \mathrm{CI}=1.928-11.502$, FDR-adjusted $p=0.004)$. In contrast, the DICER1 rs3742330 AG/RAN rs14035 CT, DROSHA rs10719 TT/RAN rs14035 CT, and RAN rs14035 CT/XPO5 AA rs11077 genotypes were more commonly observed in control participants (AOR $=0.153,95 \%$ $\mathrm{CI}=0.048-0.491$, FDR-adjusted $p=0.010 ; \mathrm{AOR}=0.318,95 \% \mathrm{CI}=0.138-0.731$, FDR-adjusted $p=0.042$; $\mathrm{AOR}=0.378,95 \% \mathrm{CI}=0.197-0.726$, FDR-adjusted $p=0.014$, respectively). 
Table 3. Genotype combination analysis for DICER1, DROSHA, RAN, and XPO5 polymorphisms in VTE patients and control subjects.

\begin{tabular}{|c|c|c|c|c|c|c|c|c|c|}
\hline Genotype & $\begin{array}{l}\text { Controls } \\
(n=300)\end{array}$ & $\begin{array}{l}\text { Total VTE } \\
(n=203)\end{array}$ & $\operatorname{AOR}(95 \% \mathrm{CI})^{\mathrm{a}}$ & $p$-value ${ }^{b}$ & $\begin{array}{c}\text { FDR-Adjusted } \\
p \text {-value }\end{array}$ & $\begin{array}{l}\text { Unprovoked VTE } \\
\quad(n=93)\end{array}$ & $\operatorname{AOR}(95 \% \mathrm{CI})^{\mathrm{a}}$ & $p$-value ${ }^{b}$ & $\begin{array}{c}\text { FDR-Adjusted } \\
p \text {-value }\end{array}$ \\
\hline \multicolumn{10}{|l|}{ DICER1/RAN } \\
\hline $\mathrm{AA}-\mathrm{CC}$ & $60(20.0)$ & $51(25.1)$ & 1.000 (reference) & & & $29(31.2)$ & 1.000 (reference) & & \\
\hline $\mathrm{AA}-\mathrm{CT}$ & 46 (15.3) & $26(12.8)$ & $0.658(0.349-1.240)$ & 0.195 & 0.520 & $12(12.9)$ & $0.446(0.192-1.038)$ & 0.061 & 0.183 \\
\hline AA-TT & $3(1.0)$ & $2(1.0)$ & $0.690(0.099-4.812)$ & 0.708 & 0.796 & $1(1.1)$ & $0.627(0.056-7.036)$ & 0.705 & 0.705 \\
\hline AG-CC & $88(29.3)$ & $68(33.5)$ & $0.936(0.567-1.545)$ & 0.796 & 0.796 & $31(33.3)$ & $0.725(0.386-1.359)$ & 0.315 & 0.473 \\
\hline GG-CC & $30(10.0)$ & $22(10.8)$ & $0.832(0.417-1.659)$ & 0.602 & 0.796 & $11(11.8)$ & $0.729(0.312-1.703)$ & 0.465 & 0.558 \\
\hline AG-CT & 45 (15.0) & $23(11.3)$ & $0.569(0.298-1.086)$ & 0.088 & 0.350 & $4(4.3)$ & $0.153(0.048-0.491)$ & 0.002 & 0.010 \\
\hline AG-TT & $4(1.3)$ & $1(0.5)$ & $0.390(0.040-3.798)$ & 0.417 & 0.796 & $0(0.0)$ & N/A & N/A & N/A \\
\hline GG-CT & $22(7.3)$ & $9(4.4)$ & $0.433(0.178-1.054)$ & 0.065 & 0.350 & $5(5.4)$ & $0.413(0.137-1.246)$ & 0.117 & 0.233 \\
\hline GG-TT & $2(0.7)$ & $1(0.5)$ & $0.610(0.045-8.209)$ & 0.710 & 0.796 & $0(0.0)$ & N/A & N/A & N/A \\
\hline \multicolumn{10}{|c|}{ DICER1/XPO5 } \\
\hline AA-AA & $95(31.7)$ & $49(24.1)$ & 1.000 (reference) & & & $26(28.0)$ & 1.000 (reference) & & \\
\hline AA-AC & $14(4.7)$ & $30(14.8)$ & $4.326(2.024-9.245)$ & 0.0002 & 0.001 & $16(17.2)$ & $4.709(1.928-11.502)$ & 0.001 & 0.004 \\
\hline AA-CC & $0(0.0)$ & $0(0.0)$ & N/A & N/A & $\mathrm{N} / \mathrm{A}$ & $0(0.0)$ & N/A & N/A & N/A \\
\hline AG-AA & $120(40.0)$ & $72(35.5)$ & $1.233(0.772-1.968)$ & 0.381 & 0.634 & $29(31.2)$ & $1.008(0.541-1.879)$ & 0.979 & 0.979 \\
\hline GG-AA & 48 (16.0) & $27(13.3)$ & $1.017(0.555-1.862)$ & 0.958 & 0.958 & $12(12.9)$ & $0.867(0.392-1.920)$ & 0.725 & 0.979 \\
\hline AG-AC & $16(5.3)$ & $20(9.9)$ & $2.387(1.112-5.125)$ & 0.026 & 0.064 & $6(6.5)$ & $1.160(0.382-3.519)$ & 0.793 & 0.979 \\
\hline AG-CC & $1(0.3)$ & $0(0.0)$ & N/A & N/A & N/A & $0(0.0)$ & N/A & N/A & N/A \\
\hline GG-AC & $6(2.0)$ & $4(2.0)$ & $1.358(0.352-5.236)$ & 0.657 & 0.821 & $3(3.2)$ & $1.994(0.436-9.116)$ & 0.374 & 0.934 \\
\hline GG-CC & $0(0.0)$ & $1(0.5)$ & N/A & $\mathrm{N} / \mathrm{A}$ & N/A & $1(1.1)$ & N/A & N/A & N/A \\
\hline \multicolumn{10}{|c|}{ DROSHA/RAN } \\
\hline TT-CC & $96(32.0)$ & $86(42.4)$ & 1.000 (reference) & & & $40(43.0)$ & 1.000 (reference) & & \\
\hline TT-CT & $61(20.3)$ & $27(13.3)$ & $0.445(0.250-0.790)$ & 0.006 & 0.040 & $10(10.8)$ & $0.318(0.138-0.731)$ & 0.007 & 0.042 \\
\hline TT-TT & $7(2.3)$ & $3(1.5)$ & $0.663(0.162-2.709)$ & 0.567 & 0.661 & $0(0.0)$ & N/A & N/A & N/A \\
\hline TC-CC & $75(25.0)$ & $45(22.2)$ & $0.692(0.427-1.121)$ & 0.135 & 0.436 & $28(30.1)$ & $0.889(0.494-1.602)$ & 0.696 & 0.914 \\
\hline CC-CC & $7(2.3)$ & $10(4.9)$ & $1.858(0.648-5.325)$ & 0.249 & 0.436 & $3(3.2)$ & $1.213(0.283-5.205)$ & 0.795 & 0.914 \\
\hline TC-CT & 46 (15.3) & $26(12.8)$ & $0.684(0.383-1.221)$ & 0.199 & 0.436 & $10(10.8)$ & $0.496(0.219-1.124)$ & 0.093 & 0.279 \\
\hline TC-TT & $2(0.7)$ & $1(0.5)$ & $0.373(0.030-4.595)$ & 0.442 & 0.618 & $1(1.1)$ & $1.152(0.090-14.704)$ & 0.914 & 0.914 \\
\hline CC-CT & $6(2.0)$ & $5(2.5)$ & $1.084(0.308-3.818)$ & 0.901 & 0.901 & $1(1.1)$ & $0.459(0.051-4.143)$ & 0.488 & 0.914 \\
\hline CC-TT & $0(0.0)$ & $0(0.0)$ & N/A & N/A & N/A & $0(0.0)$ & N/A & N/A & N/A \\
\hline \multicolumn{10}{|c|}{ DROSHA/XPO5 } \\
\hline TT-AA & $143(47.7)$ & $84(41.4)$ & 1.000 (reference) & & & $36(38.7)$ & 1.000 (reference) & & \\
\hline TT-AC & $21(7.0)$ & $31(15.3)$ & $2.385(1.239-4.590)$ & 0.009 & 0.047 & $13(14.0)$ & $2.304(1.017-5.217)$ & 0.045 & 0.136 \\
\hline TT-CC & $0(0.0)$ & $1(0.5)$ & N/A & N/A & N/A & $1(1.1)$ & N/A & N/A & $\mathrm{N} / \mathrm{A}$ \\
\hline TC-AA & $109(36.3)$ & $54(26.6)$ & $0.913(0.591-1.410)$ & 0.680 & 0.680 & $28(30.1)$ & $1.119(0.631-1.985)$ & 0.700 & 0.757 \\
\hline CC-AA & $11(3.7)$ & $10(4.9)$ & $1.776(0.700-4.504)$ & 0.226 & 0.283 & $3(3.2)$ & $1.242(0.315-4.894)$ & 0.757 & 0.757 \\
\hline TC-AC & $13(4.3)$ & $18(8.9)$ & 2.624 (1.156-5.955) & 0.021 & 0.053 & $11(11.8)$ & 3.346 (1.276-8.774) & 0.014 & 0.084 \\
\hline TC-CC & $1(0.3)$ & $0(0.0)$ & N/A & N/A & N/A & $0(0.0)$ & N/A & N/A & N/A \\
\hline CC-AC & $2(0.7)$ & $5(2.5)$ & $5.425(0.948-31.053)$ & 0.058 & 0.096 & $1(1.1)$ & $3.820(0.292-49.938)$ & 0.307 & 0.614 \\
\hline CC-CC & $0(0.0)$ & $0(0.0)$ & N/A & N/A & N/A & $0(0.0)$ & N/A & N/A & N/A \\
\hline
\end{tabular}


Table 3. Cont.

\begin{tabular}{|c|c|c|c|c|c|c|c|c|c|}
\hline Genotype & $\begin{array}{l}\text { Controls } \\
(n=300)\end{array}$ & $\begin{array}{l}\text { Total VTE } \\
(n=203)\end{array}$ & $\operatorname{AOR}(95 \% \mathrm{CI})^{\mathrm{a}}$ & $p$-value ${ }^{b}$ & $\begin{array}{c}\text { FDR-Adjusted } \\
p \text {-value }\end{array}$ & $\begin{array}{l}\text { Unprovoked VTE } \\
\quad(n=93)\end{array}$ & $\operatorname{AOR}(95 \% \mathrm{CI})^{\mathrm{a}}$ & $p$-value ${ }^{b}$ & $\begin{array}{c}\text { FDR-Adjusted } \\
p \text {-value }\end{array}$ \\
\hline \multicolumn{10}{|l|}{ RAN/XPO5 } \\
\hline CC-AA & $153(51.0)$ & $103(50.7)$ & 1.000 (reference) & & & $52(55.9)$ & 1.000 (reference) & & \\
\hline CC-AC & $25(8.3)$ & $37(18.2)$ & $2.061(1.153-3.686)$ & 0.015 & 0.045 & 18 (19.4) & 1.965 (0.971-3.975) & 0.060 & 0.120 \\
\hline CC-CC & $0(0.0)$ & $1(0.5)$ & $\mathrm{N} / \mathrm{A}$ & N/A & N/A & $1(1.1)$ & N/A & N/A & N/A \\
\hline CT-AA & $103(34.3)$ & $43(21.2)$ & $0.583(0.373-0.912)$ & 0.018 & 0.045 & $15(16.1)$ & $0.378(0.197-0.726)$ & 0.004 & 0.014 \\
\hline TT-AA & $7(2.3)$ & $2(1.0)$ & $0.431(0.085-2.179)$ & 0.309 & 0.386 & $0(0.0)$ & N/A & N/A & N/A \\
\hline CT-AC & $9(3.0)$ & $15(7.4)$ & $2.191(0.898-5.346)$ & 0.085 & 0.142 & $6(6.5)$ & $1.368(0.432-4.338)$ & 0.594 & 0.698 \\
\hline CT-CC & $1(0.3)$ & $0(0.0)$ & N/A & $\mathrm{N} / \mathrm{A}$ & $\mathrm{N} / \mathrm{A}$ & $0(0.0)$ & N/A & N/A & N/A \\
\hline TT-AC & $2(0.7)$ & $2(1.0)$ & 1.507 (0.201-11.300) & 0.690 & 0.690 & $1(1.1)$ & $1.638(0.135-19.801)$ & 0.698 & 0.698 \\
\hline TT-CC & $0(0.0)$ & $0(0.0)$ & N/A & N/A & N/A & $0(0.0)$ & N/A & N/A & N/A \\
\hline
\end{tabular}

$p$-values $<0.05$ are shown in bold. Abbreviations: AOR, adjusted odds ratio; $95 \% \mathrm{CI}, 95 \%$ confidence interval; FDR, false-positive discovery rate; VTE, venous thromboembolism. 
2.4. Genotype Combinations of miRNA Biogenesis Gene Polymorphisms

We then analyzed allele combinations of the four miRNA biogenesis genes polymorphisms in VTE patients and control subjects (Table 4). When total VTE patients were compared with controls, the C-C (RAN/XPO5), T-C-C (DROSHA/RAN/XPO5), A-T-C (DICER1/RAN/XPO5), A-T-C-C (DICER1/DROSHA/RAN/XPO5), and A-T-T-C (DICER1/DROSHA/RAN/XPO5) genotypes were significantly associated with an increased risk of VTE $(p<0.05)$. Conversely, the T-A (RAN/XPO5), T-T-A (DROSHA/RAN/XPO5), and A-T-T-A (DICER1/DROSHA/RAN/XPO5) genotypes were significantly less frequent in patients with VTE $(p<0.05)$. In patients with unprovoked VTE, the C-C (RAN/XPO5) and T-C-C (DROSHA/RAN/XPO5) genotypes were associated with an increased risk of VTE $(p<0.05)$. However, the T-A (RAN/XPO5), T-T-A (DROSHA/RAN/XPO5), A-T-T-A (DICER1/DROSHA/RAN/XPO5) genotypes were significantly less frequent in patients with VTE $(p<0.01)$. 
Table 4. Allele combination analysis of the DICER1, DROSHA, RAN, and XPO5 gene polymorphisms in VTE patients and controls.

\begin{tabular}{|c|c|c|c|c|c|c|c|c|c|}
\hline $\begin{array}{c}\text { Allele } \\
\text { Combination }\end{array}$ & $\begin{array}{l}\text { Controls } \\
(2 n=600)\end{array}$ & $\begin{array}{l}\text { Total VTE } \\
(2 n=406)\end{array}$ & OR $(95 \% \mathrm{CI})$ & $p$-value ${ }^{a}$ & $\begin{array}{c}\text { FDR-Adjusted } \\
p \text {-value }\end{array}$ & $\begin{array}{c}\text { Unprovoked VTE } \\
(2 n=186)\end{array}$ & OR $(95 \% \mathrm{CI})$ & $p$-value ${ }^{a}$ & $\begin{array}{c}\text { FDR-Adjusted } \\
p \text {-value }\end{array}$ \\
\hline \multicolumn{10}{|c|}{ DICER1/DROSHA/RAN/XPO5 } \\
\hline A-T-C-A & $184(30.6)$ & $137(33.7)$ & 1.000 (reference) & & & $74(39.9)$ & 1.000 (reference) & & \\
\hline A-T-C-C & $13(2.1)$ & $21(5.2)$ & $2.170(1.049-4.486)$ & 0.033 & 0.144 & $11(6.0)$ & $2.104(0.902-4.909)$ & 0.080 & 0.173 \\
\hline A-T-T-A & $64(10.7)$ & $16(4.0)$ & $0.336(0.186-0.606)$ & 0.0002 & 0.003 & $3(1.8)$ & $0.117(0.035-0.383)$ & $<0.0001$ & 0.001 \\
\hline A-T-T-C & $2(0.4)$ & $10(2.4)$ & $6.715(1.447-31.160)$ & 0.005 & 0.035 & $4(2.4)$ & $4.973(0.891-27.750)$ & 0.065 & 0.168 \\
\hline A-C-C-A & 71 (11.9) & $34(8.4)$ & $0.643(0.404-1.024)$ & 0.062 & 0.181 & $13(7.0)$ & $0.455(0.238-0.872)$ & 0.016 & 0.051 \\
\hline A-C-C-C & $9(1.5)$ & $13(3.2)$ & $1.940(0.806-4.670)$ & 0.182 & 0.295 & $4(2.4)$ & $1.105(0.330-3.701)$ & 1.000 & 1.000 \\
\hline A-C-T-A & $13(2.1)$ & $19(4.7)$ & $1.963(0.937-4.112)$ & 0.070 & 0.181 & $8(4.5)$ & $1.530(0.609-3.845)$ & 0.363 & 0.524 \\
\hline A-C-T-C & $0(0)$ & $0(0.0)$ & N/A & N/A & N/A & $0(0.0)$ & N/A & N/A & N/A \\
\hline G-T-C-A & $142(23.7)$ & $91(22.3)$ & $0.861(0.610-1.214)$ & 0.392 & 0.464 & $32(17.3)$ & $0.560(0.351-0.896)$ & 0.015 & 0.051 \\
\hline G-T-C-C & $10(1.6)$ & $12(2.9)$ & $1.612(0.677-3.840)$ & 0.277 & 0.361 & $7(3.7)$ & $1.741(0.638-4.746)$ & 0.274 & 0.445 \\
\hline G-T-T-A & $34(5.6)$ & $18(4.4)$ & $0.711(0.385-1.312)$ & 0.274 & 0.188 & $7(3.7)$ & $0.512(0.217-1.206)$ & 0.120 & 0.051 \\
\hline G-T-T-C & $4(0.6)$ & $0(0.0)$ & $0.149(0.008-2.794)$ & 0.141 & 0.188 & $0(0.0)$ & $0.275(0.015-5.178)$ & 0.580 & 0.051 \\
\hline G-C-C-A & $41(6.8)$ & $32(8.0)$ & $1.048(0.628-1.750)$ & 0.857 & 0.188 & $21(11.3)$ & $1.274(0.705-2.300)$ & 0.422 & 0.051 \\
\hline G-C-T-A & $14(2.3)$ & $4(0.9)$ & $0.384(0.124-1.192)$ & 0.087 & 0.188 & $0(0.0)$ & $0.085(0.005-1.451)$ & 0.014 & 0.051 \\
\hline G-C-T-C & $1(0.1)$ & $0(0.0)$ & 0.447 (0.018-11.070) & 1.000 & 0.188 & $0(0.0)$ & $0.826(0.033-20.510)$ & 1.000 & 0.051 \\
\hline \multicolumn{10}{|c|}{ DICER1/RAN/XPO5 } \\
\hline A-C-A & $254(42.3)$ & $175(43.0)$ & 1.000 (reference) & & & $89(47.6)$ & 1.000 (reference) & & \\
\hline A-C-C & $22(3.6)$ & $33(8.1)$ & $2.177(1.228-3.861)$ & 0.007 & 0.023 & $15(7.9)$ & $1.946(0.967-3.916)$ & 0.059 & 0.103 \\
\hline A-T-A & 77 (12.8) & $32(7.8)$ & $0.603(0.383-0.951)$ & 0.028 & 0.066 & $11(6.1)$ & $0.408(0.207-0.802)$ & 0.008 & 0.054 \\
\hline A-T-C & $2(0.4)$ & $11(2.7)$ & $7.983(1.747-36.470)$ & 0.002 & 0.011 & $4(2.4)$ & $5.708(1.027-31.710)$ & 0.046 & 0.103 \\
\hline G-C-A & $184(30.7)$ & $120(29.6)$ & $0.947(0.701-1.278)$ & 0.720 & 0.720 & $52(28.0)$ & $0.807(0.545-1.193)$ & 0.281 & 0.281 \\
\hline $\mathrm{G}-\mathrm{C}-\mathrm{C}$ & $9(1.5)$ & $12(3.0)$ & $1.935(0.798-4.692)$ & 0.138 & 0.193 & $8(4.2)$ & $2.537(0.950-6.778)$ & 0.088 & 0.123 \\
\hline G-T-A & $47(7.9)$ & $24(5.8)$ & $0.741(0.437-1.257)$ & 0.265 & 0.309 & $7(3.8)$ & $0.425(0.185-0.975)$ & 0.038 & 0.103 \\
\hline G-T-C & $5(0.8)$ & $0(0.0)$ & $0.132(0.007-2.401)$ & 0.085 & 0.148 & $0(0.0)$ & $0.259(0.014-4.725)$ & 0.187 & 0.218 \\
\hline \multicolumn{10}{|c|}{ DROSHA/RAN/XPO5 } \\
\hline T-C-A & $326(54.3)$ & $231(56.8)$ & 1.000 (reference) & & & $108(58.3)$ & 1.000 (reference) & & \\
\hline T-C-C & $23(3.8)$ & $32(7.8)$ & $1.963(1.120-3.444)$ & 0.017 & 0.056 & $16(8.8)$ & $2.100(1.070-4.121)$ & 0.028 & 0.098 \\
\hline T-T-A & $97(16.1)$ & $32(7.9)$ & $0.466(0.302-0.718)$ & 0.001 & 0.004 & $9(4.8)$ & $0.280(0.137-0.574)$ & 0.0002 & 0.001 \\
\hline T-T-C & $6(1)$ & $10(2.5)$ & $2.352(0.843-6.564)$ & 0.093 & 0.163 & $5(2.7)$ & $2.515(0.752-8.409)$ & 0.157 & 0.274 \\
\hline C-C-A & $112(18.7)$ & $63(15.6)$ & $0.794(0.558-1.129)$ & 0.198 & 0.277 & $33(17.5)$ & $0.889(0.570-1.388)$ & 0.606 & 0.848 \\
\hline C-C-C & $8(1.4)$ & $15(3.6)$ & $2.646(1.103-6.346)$ & 0.024 & 0.056 & $6(3.0)$ & $2.264(0.768-6.672)$ & 0.129 & 0.274 \\
\hline C-T-A & $28(4.6)$ & $24(5.9)$ & $1.210(0.684-2.141)$ & 0.513 & 0.598 & $9(4.8)$ & $0.970(0.444-2.121)$ & 0.940 & 1.000 \\
\hline C-T-C & $1(0.2)$ & $0(0.0)$ & $0.470(0.019-11.600)$ & 1.000 & 1.000 & $0(0.0)$ & $1.003(0.041-24.820)$ & 1.000 & 1.000 \\
\hline \multicolumn{10}{|l|}{ RAN/XPO5 } \\
\hline C-A & $438(73)$ & $294(72.4)$ & 1.000 (reference) & & & $141(75.7)$ & 1.000 (reference) & & \\
\hline $\mathrm{C}-\mathrm{C}$ & $31(5.2)$ & 46 (11.3) & $2.211(1.369-3.569)$ & 0.001 & 0.003 & $22(12.0)$ & $2.205(1.236-3.932)$ & 0.006 & 0.009 \\
\hline T-A & $124(20.7)$ & $56(13.8)$ & $0.673(0.475-0.953)$ & 0.025 & 0.038 & $18(9.8)$ & $0.451(0.266-0.766)$ & 0.003 & 0.008 \\
\hline $\mathrm{T}-\mathrm{C}$ & $7(1.2)$ & $10(2.5)$ & $2.128(0.801-5.656)$ & 0.121 & 0.121 & $5(2.5)$ & $2.219(0.693-7.103)$ & 0.181 & 0.181 \\
\hline
\end{tabular}

a $P$-values were calculated using Chi-square test and Fisher's exact test; significant $p$-values $<0.05$ are shown in bold. Abbreviations: OR, odds ratio; CI, confidence interval; FDR, false-positive discovery rate; OD, odds ratio; VTE, venous thromboembolism. 


\section{Discussion}

Hemostasis has the dual role of retaining blood flow and preventing inappropriate coagulation [11]. Previous studies have demonstrated that miRNAs and miRNA biogenesis genes (e.g., DICER1 and DROSHA) play important roles in vascular biology and hemostasis. For example, the platelet miRNA profiles are associated with platelet functions, miRNA biogenesis gene may alter platelet miRNA expression. When DICER1 gene, one of the miRNA biogenesis genes, was deleted, platelet miRNA profile was changed, and platelet reactivity was enhanced [37,38]. In particular, miRNA-145 is a significant factor in the prevention of thrombosis production and is present at decreased levels in VTE patients [14]. Despite the fact that the miRNA biogenesis gene haves been studied in other diseases [39-41]; however, the miRNA modulation mechanisms in VTE patients remain unclear. These miRNA biogenesis genes, including DICER1, DROSHA, RAN, and XPO5, play critical roles in miRNA production. As such, numerous other studies have found that miRNA biogenesis gene knockdown leads to a global reduction in mature miRNA levels. Those miRNAs for which decreased levels have been demonstrated are shown in Table S1 [23-27]. In this study, we analyzed the association between polymorphisms in each of the four miRNA biogenesis genes (DICER1, DROSHA, RAN, XPO5) and the risk of VTE in Korean subjects. From this analysis, we found that the RAN rs14035C > T and XPO5 rs11077A > C polymorphisms are associated with total VTE and unprovoked VTE susceptibility. Additionally, in our Genotype combination and allele combination data, we found that RAN rs14035 T allele was associated with decreased risk of VTE and XPO5 rs11077 $C$ allele was associated with increased risk of VTE [36].

RAN is small Ras-related GTP-binding protein that plays an important role in the cell cycle. Specifically, RAN functions in the transport of molecules from the nucleus to the cytoplasm or from the cytoplasm to the nucleus through the nuclear pore complex, in a GTP-dependent manner [42]. In miRNA biology, RAN associates with a protein known as XPO5 to transport pre-miRNA precursors from the nucleus to the cytoplasm. Exportin (XPO) proteins are members of the karyopherin $\beta$ family of transport factors, and XPO5 is one of the nucleo-cytoplasmic exportins. RAN, XPO5, and the pre-miRNA molecule form a complex, which functions to transport the pre-miRNA through the nuclear pore complex and into the cytoplasm. Additionally, there is a correlation between the expression of RAN and XPO5. In breast cancer, when RAN GTPase was overexpressed, XPO5 level was also significantly increased.

The XPO5 gene has been found to play a role in carcinogenesis, as it was reported that certain cancers (e.g., non-small-cell lung cancer, esophageal squamous cell carcinoma, gastric cancer, hepatocellular carcinoma, and thyroid cancer) express levels of XPO5 that are distinct from those detected in normal cells [43-48]. In particular, a number of studies have reported that XPO5 polymorphisms at the rs11077 locus affect disease development and patient survival in various cancers. For example, the rs11077AA genotype displayed a trend for high expression in ESCC tissues, and these high XPO5 expression levels were also associated with high survival rates among ESCC patients [45]. In addition, the XPO5 rs11077 polymorphism is associated with XPO5 stability and miRNA expression levels. One study reported that when a polymorphism rs11077 is present in XPO5, precursor miRNAs are trapped in the nucleus, and processing efficiency decreases. This results in reduced miRNA levels, leading to diminished miRNA target gene inhibition [27]. In another case, using a Renilla luciferase $3^{\prime}$-UTR reporter assay, the rs11077 CC genotype was shown to promote reduced Renilla expression system, and knockdown of XPO5 expression lead to reduced miRNA levels [43]. In addition, XPO5 functions to regulate expression of DICER1, another miRNA biogenesis factor that cleaves the pre-miRNA with a hairpin structure to miRNA; miRNA duplex form in the cytoplasm. Notably, it was shown that decreased levels of XPO5, lead to decreased DICER1 expression [49]. Overall, these data suggest that SNPs in miRNA biogenesis genes affect the expression of mature miRNAs and consequently, may also influence miRNA-mediated regulation.

Our data further indicate that the DROSHA rs10719T > C polymorphism is associated with the incidence of provoked VTE. DROSHA has previously been shown to play a role in vascular smooth 
muscle cell survival and angiogenesis [50], and provoked VTE results from risk factors associated with pregnancy, surgery, and hormones. Therefore, these findings suggest that in provoked VTE, DROSHA is significantly associated with vascular inflammation and recovery.

There are a number of limitations to this case-control study. For example, both the VTE patient and control sample sizes were small and the study population was comprised only of Korean individuals. We will therefore need to validate these data in other ethnic groups. In addition, although we found a significant association between the RAN rs14035 and XPO5 rs11077 polymorphisms and VTE, a proposed mechanism for the role of these polymorphisms in VTE prevalence is lacking. Thus, additional studies, potentially analyzing the effects of other miRNA biogenesis genes, will be needed to clarify the nature of the association between these polymorphisms and VTE.

\section{Materials and Methods}

\subsection{Ethics Statement}

All study protocols for this genetic analysis were reviewed and approved by The Institutional Review Board of CHA Bundang Medical Center in 10 January 2012 (IRB-number: 2005-002) and followed the recommendations of the Declaration of Helsinki (Fifth revision. 7, October, 2000). Written informed consent was obtained from all study participants prior to enrollment.

\subsection{Study Population}

A set of 203 patients with a recent ( $<6$ months) objective diagnosis of DVT or PE, who visited the CHA Bundang Medical Center (Seongnam, Korea) between March 2006 and January 2011, were enrolled in the study. This VTE group included only patients with symptomatic VTE and excluded individuals with asymptomatic VTE. VTE was classified as provoked if the patient presented with at least one of the following risk factors: recent surgery ( $<3$ months), recent trauma/fracture ( $<3$ months), immobilization ( $>7$ days), malignancy, stroke, severe medical disease, autoimmune disease, pregnancy, use of oral contraceptives, or known hypercoagulable disease. VTE was classified as unprovoked if these risk factors were absent. The 300 control group was chosen among patients visiting the CHA Bundang Health Promotion Center for periodic health examinations, who had no medical history of VTE. Vascular risk factors were assessed using medical records and laboratory data at the patient's first hospital appointment. The data included in this study consisted of age, sex, hypertension, diabetes, lipidemia. Hypertension was diagnosed when a subject had a high baseline blood pressure ( $\geq$ systolic blood pressure (SBP) $140 \mathrm{mmHg}$ or diastolic blood pressure (DBP) $90 \mathrm{mmHg}$ ) or patient had been taken anti-hypertensive medication. Diabetes mellitus was diagnosed when subject's high fasting plasma glucose level was higher than $126 \mathrm{mg} / \mathrm{dL}$ or had been taken anti-diabetic drugs. Smoking indicates current smoker at the time of the examination. Hyperlipidemia was defined as fasting serum total cholesterol of $240 \mathrm{mg} / \mathrm{dL}$ or a history of anti-hyperlipidemic agent treatment [51].

\subsection{Genotyping}

Peripheral blood samples were collected in blood collection tube and were ethylenediaminetetraacetic acid (EDTA) treated. Blood samples were centrifuged at $3000 \mathrm{rpm}$ for $15 \mathrm{~min}$, the buffy coat layer was collected, and leukocytes were separated. DNA was extracted from subject leukocytes using the G-DEX II Genomic DNA Extraction Kit (Intron Biotechnology, Seongnam, Korea), according to manufacturer instructions. We then assayed the four best-studied single nucleotide polymorphisms (SNPs) in the miRNA biogenesis genes, as determined by a literature search, which included the following 3'-UTR SNPs: DICER1 rs3742330A > G, DROSHA rs10719T > C, RAN rs14035C > T, and XPO5 rs11077A $>C$. These miRNA biogenesis gene polymorphisms were analyzed by the polymerase chain reaction-restriction fragment length polymorphism (PCR-RFLP) method, and the PCR conditions for these analyses are presented in Table S2. 


\subsection{Statistical Analysis}

Clinical characteristics of the study subjects were compared using the independent sample t-test. Associations among VTE prevalence and the four miRNA biogenesis genotypes and allele combination frequencies were estimated by analyzing the odds ratios (ORs) and $95 \%$ confidence intervals (CIs) with multivariate logistic regression and the Fisher's exact test, respectively. Adjusted ORs (AORs) for the polymorphisms were determined using multiple logistic regression analysis based on gender, age, diabetes mellitus, hypertension, hyperlipidemia, and smoking status. The genotype distribution for each polymorphism was assessed for Hardy-Weinberg equilibrium deviations, and the genotype and allele frequency differences between groups were assessed using $\chi 2$ tests. $p$-values $<0.05$ were considered to be statistically significant. HAPSTAT software, version 3.0 (University of North Carolina, Chapel Hill, NC, USA) (www.bios.unc.edu/Elin/hapstat/) was used to estimate haplotype frequencies for polymorphisms that were determined by multifactor dimensionality reduction analyses to have strong synergistic effects. Statistical analyses to measure the association between DICER1, DROSHA, RAN, and XPO5 polymorphisms and VTE prevalence were performed using MedCalc, version 18.9 (MedCalc Software, Mariakerke, Belgium) and GraphPad Prism, version 4.0 (GraphPad, San Diego, CA, USA) software. The false-positive discovery rate (FDR) correction was used to adjust for multiple comparison tests, and associations with FDR-adjusted $p$-values $<0.05$ were considered to be significant.

\section{Conclusions}

In this study, we have identified an association between susceptibility to VTE and polymorphisms in the miRNA biogenesis genes, RAN rs14035C $>\mathrm{T}$ and XPO5 rs11077A $>\mathrm{C}$. These findings may provide the basis for continued research efforts focusing on the roles of RAN and XPO5 in hemostasis and thrombus development. We propose that the RAN rs14035 and XPO5 rs11077 polymorphisms influence miRNA biosynthesis, and therefore, affect miRNA post-transcriptional regulation during hemostasis (i.e., platelet biogenesis, coagulation, and anticoagulation) and thrombus formation. However, the mechanisms underlying these relationships remain to be elucidated in future research.

Supplementary Materials: The following are available online at http://www.mdpi.com/1422-0067/20/15/3771/s1.

Author Contributions: Conceptualization, E.J.K., E.J.K. and N.K.K.; Methodology, E.J.K. and C.S.R.; Formal Analysis, J.H.S. and H.S.P.; Investigation, E.J.K. and J.O.K.; Resources, E.J.K., J.H.S., J.O., and S.Y.C.; Data Curation, J.O.K., H.S.P., and C.S.R.; Writing-Original Draft Preparation, E.J.K. and J.O.K. Writing-Review \& Editing, E.J.K., S.Y.C, D.O., and N.K.K.; Supervision, D.O. and N.K.K.; Project Administration, D.O. and N.K.K.; Funding Acquisition, N.K.K.

Acknowledgments: This study was partially supported by a grant of the Korea Health Technology R\&D Project through the Korea Health Industry Development Institute (KHIDI), funded by the Ministry of Health \& Welfare, Republic of Korea (HI18C19990200) and partially supported by National Research Foundation of Korea (NRF) funded by the Ministry of Education, Science and Technology (2017R1D1A1B03030110).

Conflicts of Interest: The authors declare no conflict of interest.

\section{References}

1. ISCfWT, D. Thrombosis: A major contributor to the global disease burden. J. Thromb. Haemost. 2014, 12, 1580-1590.

2. Di Nisio, M.; van Es, N.; Büller, H.R. Deep vein thrombosis and pulmonary embolism. Lancet 2016, 388, 3060-3073. [CrossRef]

3. Lee, J.S.; Moon, T.; Kim, T.H.; Kim, S.Y.; Choi, J.Y.; Lee, K.B.; Kwon, Y.J.; Song, S.H.; Kim, S.H.; Kim, H.O.; et al. Deep Vein Thrombosis in Patients with Pulmonary Embolism: Prevalance, Clinical Significance and Outcome. Vasc. Specialist Int. 2016, 32, 166-174. [CrossRef]

4. Hong, J.; Lee, J.H.; Yhim, H.Y.; Choi, W.I.; Bang, S.M.; Lee, H.; Oh, D. Incidence of venous thromboembolism in Korea from 2009 to 2013. PLoS ONE 2018, 13, e0191897. [CrossRef] 
5. Nielsen, J.D. The incidence of pulmonary embolism during deep vein thrombosis. Phlebology 2013, 28, 29-33. [CrossRef]

6. Kearon, C.; Ageno, W.; Cannegieter, S.C.; Cosmi, B.; Geersing, G.J.; Kyrle, P.A.; Subcommittees on Control of Anticoagulation, and Predictive and Diagnostic Variables in Thrombotic Disease. Categorization of patients as having provoked or unprovoked venous thromboembolism: Guidance from the SSC of ISTH. J. Thromb. Haemost. 2016, 14, 1480-1483. [CrossRef]

7. Anderson, F.A.J.; Spencer, F.A. Risk factors for venous thromboembolism. Circulation 2003, 107, I-9-I-16. [CrossRef]

8. Hollenhorst, M.A.; Battinelli, E.M. Thrombosis, Hypercoagulable States, and Anticoagulants. Prim. Care 2016, 43, 619-635. [CrossRef]

9. Kline, J.A. Diagnosis and Exclusion of Pulmonary Embolism. Thromb. Res. 2018, 163, 207-220. [CrossRef]

10. Wells, P.; Anderson, D. The diagnosis and treatment of venous thromboembolism. Hematol. Am. Soc. Hematol. Educ. Program 2013, 1, 457-463. [CrossRef]

11. Teruel-Montoya, R.; Rosendaal, F.R.; Martinez, C. MicroRNAs in hemostasis. J. Thromb. Haemost. 2015, 13, 170-181. [CrossRef]

12. Chen, L.J.; Yang, L.; Cheng, X.; Xue, Y.K.; Chen, L.B. Overexpression of miR-24 Is Involved in the Formation of Hypocoagulation State after Severe Trauma by Inhibiting the Synthesis of Coagulation Factor X. Dis. Markers 2017, 6, 3649693. [CrossRef]

13. Wang, W.; Zhu, X.; Du, X.; Xu, A.; Yuan, X.; Zhan, Y.; Liu, M.; Wang, S. MiR-150 promotes angiogensis and proliferation of endothelial progenitor cells in deep venous thrombosis by targeting SRCIN1. Microvasc. Res. 2018, 123, 35-41. [CrossRef]

14. Sahu, A.; Jha, P.K.; Prabhakar, A.; Singh, H.D.; Gupta, N.; Chatterjee, T.; Tyagi, T.; Sharma, S.; Kumari, B.; Singh, S.; et al. MicroRNA-145 Impedes Thrombus Formation via Targeting Tissue Factor in Venous Thrombosis. EBioMedicine 2017, 26, 175-186. [CrossRef]

15. Yamakuchi, M. MicroRNAs in Vascular Biology. Int. J. Vasc. Med. 2012, 2012, 794898. [CrossRef]

16. Elgheznawy, A.; Fleming, I. Platelet-Enriched MicroRNAs and Cardiovascular Homeostasis. Antioxid. Redox Signal. 2018, 29, 902-921. [CrossRef]

17. Tsunetsugu-Yokota, Y.; Yamamoto, T. Mammalian MicroRNAs: Post-Transcriptional Gene Regulation in RNA Virus Infection and Therapeutic Applications. Front. Microbiol. 2010, 1, 108. [CrossRef]

18. Wahid, F.; Shehzad, A.; Khan, T.; Kim, Y.Y. MicroRNAs: Synthesis, mechanism, function, and recent clinical trials. Biochim. Biophys. Acta 2010, 1803, 1231-1243. [CrossRef]

19. Chekulaeva, M.; Filipowicz, W. Mechanisms of miRNA-mediated post-transcriptional regulation in animal cells. Curr. Opin. Cell Biol. 2009, 21, 452-460. [CrossRef]

20. Hartig, S.M.; Hamilton, M.P.; Bader, D.A.; McGuire, S.E. The miRNA Interactome in Metabolic Homeostasis. Trends Endocrinol. Metab. 2015, 26, 733-745. [CrossRef]

21. Gascon, E.; Gao, F.B. Cause or Effect: Misregulation of microRNA Pathways in Neurodegeneration. Front. Neurosci. 2012, 6, 48. [CrossRef]

22. Lee, S.; Choi, E.; Cha, M.-J.; Hwang, K.-C. Implications of MicroRNAs in the Vascular Homeostasis and Remodeling. Muscle Cell Tissue 2015.

23. Kim, Y.K.; Kim, B.; Kim, V.N. Re-evaluation of the roles of DROSHA, Export in 5, and DICER in microRNA biogenesis. Proc. Natl. Acad. Sci. USA 2016, 113, E1881-E1889. [CrossRef]

24. Fan, M.; Krutilina, R.; Sun, J.; Sethuraman, A.; Yang, C.H.; Wu, Z.H.; Yue, J.; Pfeffer, L.M. Comprehensive analysis of microRNA (miRNA) targets in breast cancer cells. J. Biol. Chem. 2013, 288, 27480-27493. [CrossRef]

25. Goncalves, I.; Brecht, J.; Thelen, M.P.; Rehorst, W.A.; Peters, M.; Lee, H.J.; Motameny, S.; Torres-Benito, L.; Ebrahimi-Fakhari, D.; Kononenko, N.L.; et al. Neuronal activity regulates DROSHA via autophagy in spinal muscular atrophy. Sci. Rep. 2018, 8, 7907. [CrossRef]

26. Dattilo, V.; D'Antona, L.; Talarico, C.; Capula, M.; Catalogna, G.; Iuliano, R.; Schenone, S.; Roperto, S.; Bianco, C.; Perrotti, N.; et al. SGK1 affects RAN/RANBP1/RANGAP1 via SP1 to play a critical role in pre-miRNA nuclear export: A new route of epigenomic regulation. Sci Rep. 2017, 7, 45361. [CrossRef]

27. Ott, C.A.; Linck, L.; Kremmer, E.; Meister, G.; Bosserhoff, A.K. Induction of exportin-5 expression during melanoma development supports the cellular behavior of human malignant melanoma cells. Oncotarget 2016, 7, 62292-62304. [CrossRef] 
28. Gross, T.J.; Powers, L.S.; Boudreau, R.L.; Brink, B.; Reisetter, A.; Goel, K.; Gerke, A.K.; Hassan, I.H.; Monick, M.M. A microRNA processing defect in smokers' macrophages is linked to SUMOylation of the endonuclease DICER. J. Biol. Chem. 2014, 289, 12823-12834. [CrossRef]

29. Wu, Q.; Song, R.; Ortogero, N.; Zheng, H.; Evanoff, R.; Small, C.L.; Griswold, M.D.; Namekawa, S.H.; Royo, H.; Turner, J.M.; et al. The RNase III enzyme DROSHA is essential for microRNA production and spermatogenesis. J. Biol. Chem. 2012, 287, 25173-25190. [CrossRef]

30. Sun, H.L.; Cui, R.; Zhou, J.; Teng, K.Y.; Hsiao, Y.H.; Nakanishi, K.; Fassan, M.; Luo, Z.; Shi, G.; Tili, E.; et al. ERK Activation Globally Downregulates miRNAs through Phosphorylating Exportin-5. Cancer Cell 2016, 30, 723-736. [CrossRef]

31. Banerjee, J.; Nema, V.; Dhas, Y.; Mishra, N. Role of MicroRNAs in Type 2 Diabetes and Associated Vascular Complications. Biochimie 2017, 139, 9-19. [CrossRef]

32. Shi, L.; Liao, J.; Liu, B.; Zeng, F.; Zhang, L. Mechanisms and therapeutic potential of microRNAs in hypertension. Drug Discov. Today 2015, 20, 1188-1204. [CrossRef]

33. Momtazi, A.A.; Banach, M.; Pirro, M.; Stein, E.A.; Sahebkar, A. MicroRNAs: New Therapeutic Targets for Familial Hypercholesterolemia? Clin. Rev. Allergy Immunol. 2018, 54, 224-233. [CrossRef]

34. Kim, J.O.; Bae, J.; Kim, J.; Oh, S.H.; An, H.J.; Han, I.B.; Oh, D.; Kim, O.J.; Kim, N.K. Association of MicroRNA Biogenesis Genes Polymorphisms with Ischemic Stroke Susceptibility and Post-Stroke Mortality. J. Stroke 2018, 20, 110-121. [CrossRef]

35. Fiorenza, A.; Barco, A. Role of Dicer and the miRNA system in neuronal plasticity and brain function. Neurobiol. Learn. Mem. 2016, 135, 3-12. [CrossRef]

36. Vaidyanathan, S.; Thangavelu, P.U.; Duijf, P.H. Overexpression of Ran GTPase Components Regulating Nuclear Export, but not Mitotic Spindle Assembly, Marks Chromosome Instability and Poor Prognosis in Breast Cancer. Target. Oncol. 2016, 11, 677-686. [CrossRef]

37. Rowley, J.W.; Chappaz, S.; Corduan, A.; Chong, M.M.; Campbell, R.; Khoury, A.; Manne, B.K.; Wurtzel, J.G.; Michael, J.V.; Goldfinger, L.E.; et al. Dicer1-mediated miRNA processing shapes the mRNA profile and function of murine platelets. Blood 2016, 127, 1743-1751. [CrossRef]

38. Nagalla, S.; Shaw, C.; Kong, X.; Kondkar, A.A.; Edelstein, L.C.; Ma, L.; Chen, J.; McKnight, G.S.; Lopez, J.A.; Yang, L.; et al. Platelet microRNA-mRNA coexpression profiles correlate with platelet reactivity. Blood 2011, 117, 5189-5197. [CrossRef]

39. Gorucu Yilmaz, S.; Erdal, M.E.; Avci Ozge, A.; Sungur, M.A. SNP Variation in MicroRNA Biogenesis Pathway Genes as a New Innovation Strategy for Alzheimer Disease Diagnostics: A Study of 10 Candidate Genes in an Understudied Population From the Eastern Mediterranean. Alzheimer Dis. Assoc. Disord. 2016, 30, $203-209$. [CrossRef]

40. Chen, J.; Qin, Z.; Pan, S.; Jiang, J.; Liu, L.; Liu, J.; Chen, X.; Hu, Z.; Shen, H. Genetic variants in RAN, DICER and HIWI of microRNA biogenesis genes and risk of cervical carcinoma in a Chinese population. Chin. J. Cancer Res. 2013, 25, 565-571.

41. Moghbelinejad, S.; Najafipour, R.; Momeni, A. Association of rs1057035polymorphism in microRNA biogenesis pathway gene (DICER1) with azoospermia among Iranian population. Genes Genomics 2018, 40, 17-24. [CrossRef]

42. Osuch-Wojcikiewicz, E.; Bruzgielewicz, A.; Niemczyk, K.; Sieniawska-Buccella, O.; Nowak, A.; Walczak, A.; Majsterek, I. Association of Polymorphic Variants of miRNA Processing Genes with Larynx Cancer Risk in a Polish Population. Biomed. Res. Int. 2015, 2015, 298378. [CrossRef]

43. Ding, C.; Li, C.; Wang, H.; Li, B.; Guo, Z. A miR-SNP of the XPO5 gene is associated with advanced non-small-cell lung cancer. Onco. Targets Ther. 2013, 6, 877-881.

44. Geng, J.Q.; Wang, X.C.; Li, L.F.; Zhao, J.; Wu, S.; Yu, G.P.; Zhu, K.J. MicroRNA-related single-nucleotide polymorphism of XPO5 is strongly correlated with the prognosis and chemotherapy response in advanced non-small-cell lung cancer patients. Tumour. Biol. 2016, 37, 2257-2265. [CrossRef]

45. Wang, C.; Dong, H.; Fan, H.; Wu, J.; Wang, G. Genetic polymorphisms of microRNA machinery genes predict overall survival of esophageal squamous carcinoma. J. Clin. Lab. Anal. 2018, 32, e22170. [CrossRef]

46. Liao, Y.; Liao, Y.; Li, J.; Liu, L.; Li, J.; Wan, Y.; Peng, L. Genetic variants in miRNA machinery genes associated with clinicopathological characteristics and outcomes of gastric cancer patients. Int. J. Biol. Markers 2018, 33, 301-307. [CrossRef]

47. Liu, S.; An, J.; Lin, J.; Liu, Y.; Bao, L.; Zhang, W.; Zhao, J.J. Single nucleotide polymorphisms of microRNA processing machinery genes and outcome of hepatocellular carcinoma. PLoS ONE 2014, 9, e92791. [CrossRef] 
48. Wen, J.; Gao, Q.; Wang, N.; Zhang, W.; Cao, K.; Zhang, Q.; Chen, S.; Shi, L. Association of microRNA-related gene XPO5 rs11077 polymorphism with susceptibility to thyroid cancer. Medicine (Baltimore) 2017, 96, e6351. [CrossRef]

49. Bennasser, Y.; Chable-Bessia, C.; Triboulet, R.; Gibbings, D.; Gwizdek, C.; Dargemont, C.; Kremer, E.J.; Voinnet, O.; Benkirane, M. Competition for XPO5 binding between Dicer mRNA, pre-miRNA and viral RNA regulates human Dicer levels. Nat. Struct. Mol. Biol. 2011, 18, 323-327. [CrossRef]

50. Fan, P.; Chen, Z.; Tian, P.; Liu, W.; Jiao, Y.; Xue, Y.; Bhattacharya, A.; Wu, J.; Lu, M.; Guo, Y.; et al. miRNA biogenesis enzyme Drosha is required for vascular smooth muscle cell survival. PLoS ONE 2013, 8, e60888. [CrossRef]

51. Kim, M.H.; Moon, J.S.; Park, S.Y.; An, S.A.; Kim, O.J.; Kim, N.K.; Oh, S.H. Different risk factor profiles between silent brain infarction and symptomatic lacunar infarction. Eur. Neurol. 2011, 65, 250-256. [CrossRef]

(C) 2019 by the authors. Licensee MDPI, Basel, Switzerland. This article is an open access article distributed under the terms and conditions of the Creative Commons Attribution (CC BY) license (http://creativecommons.org/licenses/by/4.0/). 